\title{
Quantitative symmetry determination and symmetry mapping using convergent beam electron diffraction technique
}

\author{
Kyou-Hyun $\mathrm{Kim}^{1}$, Jian-Min Zuo ${ }^{2,3}$
}

${ }^{1}$ Advanced Process and Materials R\&BD Group, Korea Institute of Industrial Technology, Incheon, 406-840, Korea

${ }^{2}$ Department of Materials Science and Engineering, University of Illinois at Urbana-Champaign, Urbana, Illinois 61801, USA

${ }^{3}$ Frederick Seitz Materials Research Laboratory, University of Illinois at Urbana-Champaign, Urbana, Illinois 61801, USA

The symmetry recorded in convergent beam electron diffraction (CBED) patterns is in general determined by direct visual inspection [1], which does not provide uniform measurement. Furthermore, experimental CBED patterns are often noisy and deviate from the ideal symmetry because of the sample geometry and defects. Thus, the imperfection in experimental CBED patterns can lead to uncertainty in the symmetry determination [2]. Here, we propose a symmetry quantification method for CBED patterns using the profile $R$-factor $\left(R_{p}\right)[3,4]$ and the normalized cross-correlation coefficient $(\gamma)$ [5]. We have also developed computer algorithms to automate these procedures. We demonstrate that the method proposed here is highly effective and provides a more precise way to determine the symmetry in CBED patterns. The symmetry quantification method can be also combined with a scanning electron diffraction technique for symmetry mapping [6].

Figure 1 shows the image processing procedures for mirror symmetry quantification. First, the symmetry related two diffraction discs $\left(\mathrm{A}, \mathrm{A}^{\prime}\right)$ are selected about the mirror plane (yellow line) as shown in Fig. 1(a). The template $A$ is used as the reference motif so that the symmetry element is calculated by comparing with template A'. The template A and A' are first aligned (Fig. 1(c)), and A' is flipped horizontally to obtain a mirror image (A'm, Fig. 1(h)). For the rotational operation, the template $A^{\prime}$ is simply rotated by $360 / \mathrm{n}^{\circ}$ with respect to $\mathrm{n}$-fold rotation. The circular mask (Figs. 1(d) and (i)) is finally used to remove areas affected by CBED disk edge and to obtain the final templates (Figs. 1(e) and (j)). Then, we applied the profile $R$-factor $\left(R_{p}\right)$ and the normalized cross-correlation coefficient $(\gamma)$ to quantify the similarity between $\mathrm{A}\left(=\mathrm{I}_{\mathrm{A}}(\mathrm{x}, \mathrm{y})\right)$ and $\mathrm{A}_{\mathrm{m}}\left(=\mathrm{I}_{\mathrm{B}}(\mathrm{x}, \mathrm{y})\right)$ as given in Eqs. (1) and (2).

$$
\text { (1) } R_{p}=\sqrt{\frac{\sum\left\{I_{B}(x, y)-I_{A}(x, y)\right\}^{2}}{\sum I_{A}(x, y)^{2}}}, \quad \text { (2) } \gamma=\frac{\sum_{x, y}\left\{\left[I_{A}(x, y)-\overline{I_{A}}\right] \cdot\left[I_{B}(x, y)-\overline{I_{B}}\right]\right\}}{\sqrt{\left\{\sum_{x, y}\left[I_{A}(x, y)-\overline{I_{A}}\right]^{2}\right\} \cdot\left\{\sum_{x, y}\left[I_{B}(x, y)-\overline{I_{B}}\right]^{2}\right\}}}
$$

The symmetry quantification algorithm was then combined with the scanning electron diffraction technique for symmetry mapping. Figure 2(a) shows a typical Bragg diffraction contrast for a stacking fault in a strained Si crystal. The mirror selected for quantification is along the yellow line as indicated in Fig. 2(b). The symmetry variation was mapped using the $\mathrm{A} / \mathrm{A}^{\prime}, \mathrm{B} / \mathrm{B}$ ' and $\mathrm{C} / \mathrm{C}^{\prime}$ disk pairs, and the symmetry distribution was mapped for $152 \times 72 \mathrm{~nm}^{2}$ from Fig. 2(a). Figures 2(c) and (e) are the calculated symmetry maps for $R_{p}$ and $\gamma$, respectively. The grid in the symmetry maps becomes bright as the symmetry of the investigated grid matches the selected symmetry (i.e., mirror). In both maps, the dark contrast indicates symmetry breaking from the selected mirror symmetry. For example, the profile of $R_{p}$ and $\gamma$ values were selected along the line indicated in Fig. 2(a) and plotted in Figs. 2(d) and (f), respectively. In the area of stacking fault, the $R_{p}$ rapidly increases from 0.19 to 0.79 , and the $\gamma$ drops 
significantly from 0.98 to 0.18 . Thus, the symmetry breaking is detected across the stacking fault and near the stacking fault. The details of symmetry quantification and mapping can be found in the reference [7].

We have proposed a symmetry quantification method by using $R_{p}$ and $\gamma$. The result for the Si single crystal shows that $R_{p}$ with $\sim 0.1$ and $\gamma$ with $\sim 0.98$ can be used to determine a symmetrical pattern. In addition, the Si single crystal has a constant symmetry over the scanning area while the strained $\mathrm{Si}$ sample shows large symmetry variation over a stacking fault. We believe that this study provides a powerful tool for symmetry study in real materials.

\section{References:}

[1] T. Hahn, "International tables for crystallography", Volume A, ed. 5 (Springer).

[2] K.-H. Kim, David A. Payne and J.-M. Zuo, Phys. Rev. B 86 (2012), 184113.

[3] E. Jansen et al, J. Appl. Cryst. 27 (1994), 492-496.

[4] B.H. Toby, Powder Diffraction 21 (2006), 67-70.

[5] J.P. Lewis, Vision Interface 95 (1995), 120-123.

[6] J. Tao et al, Phys. Rev. Lett 103 (2009).

[7] K.-H. Kim and J. -M. Zuo, Ultramicroscopy 124 (2013), 71.

[8] This work was supported by DOE (DEFG02-01ER45923). We thank Jerome Pacaud for providing the strained silicon sample.
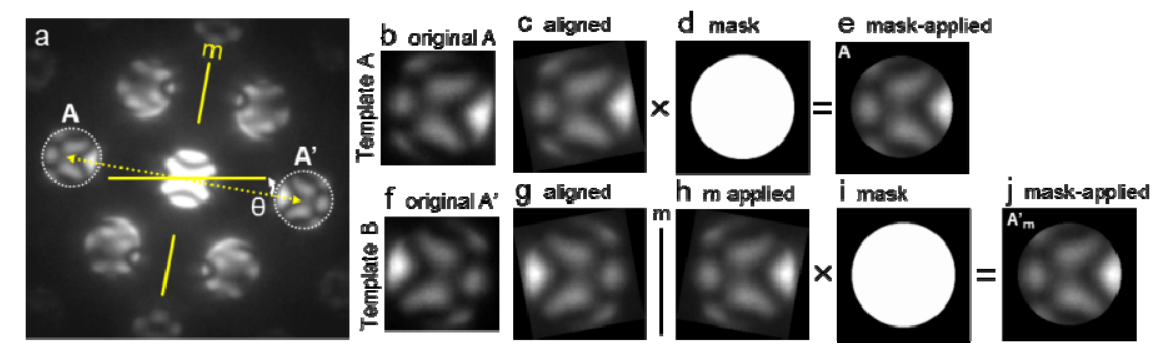

Figure 1. Image processing procedures used for mirror symmetry quantification. Two diffraction discs related by mirror are selected as indicated by the dotted circles $A$ and $A^{\prime}$ in the (a). Each disc is then processed to give two templates $\mathrm{A}$ and $\mathrm{A}^{\prime} \mathrm{m}$ as shown above.
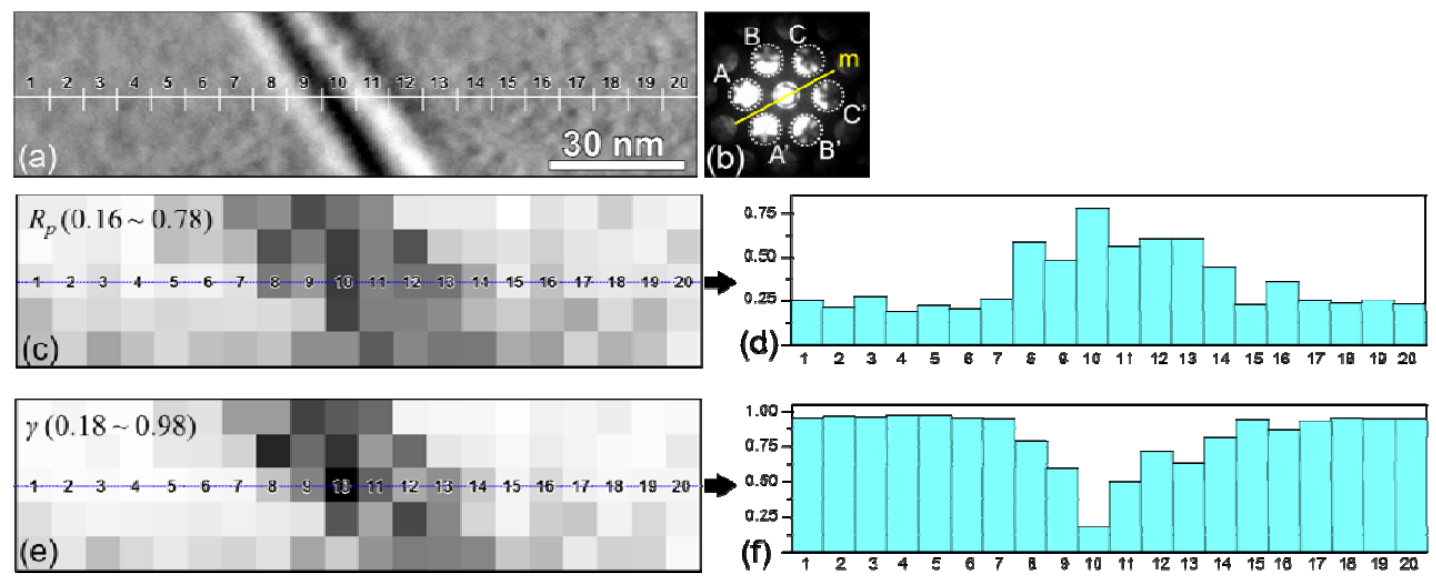

Figure 2. (a) A bright field image of strained silicon showing a stacking fault, and (b) the selected CBED pattern. The symmetry maps for $R_{p}$ and $\gamma$ are shown in Figs. 7(c) and (e), respectively. The (d) and (f) show the $R_{p}$ and $\gamma$ profile across the stacking fault along the line indicated in the (a). 